\title{
Phase and Vortex Correlations in Superconducting Josephson-Junction Arrays at Irrational Magnetic Frustration
}

\author{
Enzo Granato \\ Laboratório Associado de Sensores e Materiais, Instituto Nacional de Pesquisas Espaciais, \\ 12245-970 São José dos Campos, SP Brazil \\ (Received 9 January 2008; published 11 July 2008)
}

\begin{abstract}
Phase coherence and vortex order in a Josephson-junction array at irrational frustration are studied by extensive Monte Carlo simulations using the parallel-tempering method. A scaling analysis of the correlation length of phase variables in the full equilibrated system shows that the critical temperature vanishes with a power-law divergent correlation length and critical exponent $\nu_{\mathrm{ph}}$, in agreement with recent results from resistivity scaling analysis. A similar scaling analysis for vortex variables reveals a different critical exponent $\nu_{v}$, suggesting that there are two distinct correlation lengths associated with a decoupled zero-temperature phase transition.
\end{abstract}

PACS numbers: 74.81.Fa, 74.25.Op, 74.25.Qt

Josephson-junction arrays at irrational frustration have attracted considerable interest, both experimentally and theoretically, as a possible physical realization of a twodimensional vortex glass or a pinned incommensurate vortex lattice, without intrinsic disorder. Frustration without disorder can, in principle, be introduced by applying an external magnetic field in a perfect periodic array of weakly coupled superconducting grains [1-3] and similarly in superconducting wire networks $[4,5]$. The frustration parameter $f$, the number of flux quanta per plaquette, sets the average density of the vortex lattice and can be tuned by varying the strength of the external field [6]. At rational $f$, the ground state is a pinned vortex lattice commensurate with the array leading to discrete symmetries in addition to the continuous $U(1)$ symmetry of the phase variables characterizing the superconducting order parameter. The phase transitions and resistive behavior of the array are only reasonably well understood for simple rational $f$. At irrational $f$, however, when the vortex lattice is incommensurate with the array, the nature of both the equilibrium phase transition and the low-temperature state in the thermodynamic limit remains unclear.

We consider a Josephson-junction array on a square lattice described by the Hamiltonian [6]

$$
H=-J \sum_{\langle i j\rangle} \cos \left(\theta_{i}-\theta_{j}-A_{i j}\right) .
$$

$\theta_{i}$ is the phase of the local superconducting order parameter, $J>0$ is the uniform Josephson-junction coupling, and $A_{i j}$ is constrained to be $\sum_{i j} A_{i j}=2 \pi f$ around each plaquette, where $f$ is an irrational number $f=(3-\sqrt{5}) / 2$, related to the golden ratio $\Phi=(1+\sqrt{5}) / 2$ as $f=1-$ $1 / \Phi$.

In early Monte Carlo (MC) simulations [7], the ground state was found to consist of a disordered vortex pattern, and a possible superconducting (vortex-glass) transition at a finite temperature $T_{g} \sim 0.25$ was proposed from the behavior of the specific heat. On the other hand, some arguments suggested that the critical temperature $T_{c}$ should vanish $[6,8]$. Simulations of the current-voltage scaling found indeed a behavior consistent with a $T_{c}=0$ phase transition [9] similar to the vortex-glass model in two dimensions $[10,11]$ but with a different correlation-length critical exponent $\nu \sim 1$. However, since resistivity scaling probes mainly phase coherence, the behavior of the vortex correlation still remained to be investigated. Simulations of the relaxation dynamics by Kim and Lee [12] of the vortex variables found a behavior analogous to supercooled liquids [12] with a dynamic crossover temperature close to the apparent $T_{g}$ observed earlier in the specific heat [7]. MC simulations [13], using the vortex representation for rational $f$ converging to the irrational frustration, suggested two phase transitions at finite temperatures: a firstorder transition to an ordered vortex structure weakly dependent on $f$ and a phase-coherence transition at much lower temperatures varying significantly with $f$. The results were in qualitative agreement with other MC simulations using the phase representation [14], but different ground states were found, and the first-order behavior was also sensitive to the boundary conditions.

More recently, a study of the finite-size behavior of the specific heat and relaxation time in the phase representation found an intrinsic finite-size effect [15]. The scaling analysis confirmed the $T_{c}=0$ transition scenario with $\nu$ consistent with the earlier estimate from current-voltage scaling [9]. Other simulations agree that, below some temperature, relaxation processes become very slow. Very recently, the $T_{c}=0$ scenario received further support from improved calculations using a driven $\mathrm{MC}$ dynamics [16]. On the other hand, an analysis of the low-temperature configurations for $f$ close to the irrational value from MC simulations in the vortex representation [17] suggested two transitions, consistent with earlier work [13].

In view of these conflicting results, it is important to determine the true equilibrium behavior using methods that ensure full equilibration of the system and obtain the 
critical behavior directly from the phase and vortexcorrelation lengths within the same framework.

In this work we study phase coherence and vortex order at irrational frustration by extensive MC simulations, using the parallel-tempering method (exchange MC method) [18] to obtain equilibrium configurations of the system. This method has been shown to reduce significantly the long equilibration times in glassy systems [18-20] and supercooled liquids [21]. To study the equilibrium phase transitions, we use numerical data in the temperature regime in which full equilibration can be ensured and employ a scaling analysis to extrapolate to the low-temperature and large-system limits. Since finite-size scaling of the correlation length is currently one of the most reliable approaches to demonstrate the existence of an equilibrium finite-temperature transition for glassy systems [19,20], we use this analysis for the phase and vortex variables. The results indicate that the critical temperature for phase coherence vanishes $\left(T_{c}=0\right)$ with a power-law divergent correlation length and corresponding critical exponent $\nu_{\mathrm{ph}}$, in agreement with recent results from resistivity scaling [16]. Although a first-order vortex transition at finite temperatures cannot be ruled out, a similar scaling analysis for vortex variables is also consistent with $T_{c}=0$ but with a different exponent $\nu_{v}$. These different exponents suggest the interesting scenario where there are two distinct correlation lengths associated with a decoupled $T_{c}=0$ phase transition.

In the numerical simulations, we use periodic boundary conditions on lattices of linear sizes $L$ and corresponding rational approximations $\Phi_{n}=F_{n+1} / F_{n}$, where $F_{n}$ are Fibonacci numbers $(5,8,13,21,34,55)$, with $L=F_{n}$. Additional calculations using periodic (fluctuating twist) boundary conditions [16] or the exact value of $f$ did not change the results.

To study phase coherence, we consider the overlap order parameter [22] of the phase variables defined as $q_{\mathrm{ph}}(j)=$ $\exp \left(i \theta_{j}^{1}-i \theta_{j}^{2}\right)$, where 1 and 2 denote two thermally independent copies of the system with the same parameters $J$ and $f$. At high temperatures, where each copy is thermally disordered, the correlation function $C_{\mathrm{ph}}(r)=$ $\frac{1}{L^{2}} \sum_{j}\left\langle q_{\mathrm{ph}}(j) q_{\mathrm{ph}}(j+r)\right\rangle$ is short-ranged, decaying exponentially with $r$, while at low temperatures it is long-ranged if an ordered phase exists, including the possibility of a glassy-ordered phase. The corresponding correlation length in the finite-size system $\xi_{\text {ph }}$ can be obtained from a second moment calculation using the correlation function as [19]

$$
\xi_{\mathrm{ph}}(L)=\frac{1}{2 \sin \left(k_{o} / 2\right)}\left(\frac{S_{\mathrm{ph}}(0)}{S_{\mathrm{ph}}\left(k_{o}\right)}-1\right)^{1 / 2},
$$

where $S_{\mathrm{ph}}(k)$ is the Fourier transform of $C_{\mathrm{ph}}(r)$ and $k_{o}=$ $\left(\frac{2 \pi}{L}, 0\right)$ is the smallest wave vector in the finite system. The same expressions are used to determine the correlation length for vortex variables $\xi_{v}$ in terms of the vorticity $v_{p}$, replacing $q_{\mathrm{ph}}$ by $q_{v}(p)=v_{p}^{1} v_{p}^{2}$. The vorticity is defined as $v_{p}=\sum_{i j}\left(\theta_{i}-\theta_{j}-A_{i j}\right) / 2 \pi$ and is a measure of the local vortex density, where the summation is taken over the elementary plaquette $p$ of the lattice and the gaugeinvariant phase difference is restricted to the interval $[-\pi, \pi]$.

We use the parallel-tempering method [18] to obtain the equilibrium configurations. Many replicas of the system with different temperatures are simulated simultaneously, and the corresponding configurations are allowed to be exchanged with a probability satisfying detailed balance. The exchange process allows the configurations of the system to explore the temperature space, being cooled down and warmed up, and the system can escape more easily from metastable minima at low temperatures. With this method, full equilibration can be ensured in finite-size systems $[18,20,21]$. Without the replica exchange step, the method reduces to conventional MC simulations at different temperatures. We performed MC simulations using the heat-bath algorithm for each replica, simultaneously and independently, for a few MC passes. Then an exchange of pairs of replica configurations at temperatures $T_{i}$ and $T_{j}$ and energies $E_{i}$ and $E_{j}$ is attempted with probability $\min (1, \exp (-\Delta))$, where $\Delta=\left(1 / T_{i}-1 / T_{j}\right)\left(E_{j}-E_{i}\right)$, using the Metropolis scheme. The equilibration time to reach thermal equilibrium can be measured as the average number of MC passes required for each replica to travel over the whole temperature range. We used typically $4 \times 10^{6}$ MC passes for equilibration with up to 100 replicas and an equal number of MC passes for calculations of average quantities. Nevertheless, for the largest system sizes $L=$ 21-55, equilibration was possible only for temperatures above $T_{f} \sim 0.145$. This can be inferred from the time evolution, in the temperature space, of a replica initially at the highest temperature, as shown in Fig. 1. The replica configuration starting at $T=0.3$ is able to explore only the temperature space containing 100 temperatures down to $T_{f} \sim 0.145$. Below this temperature, the replicas cannot be warmed up and cooled down. Thus, $T_{f}$ can be regarded as a freezing temperature, below which the system remains

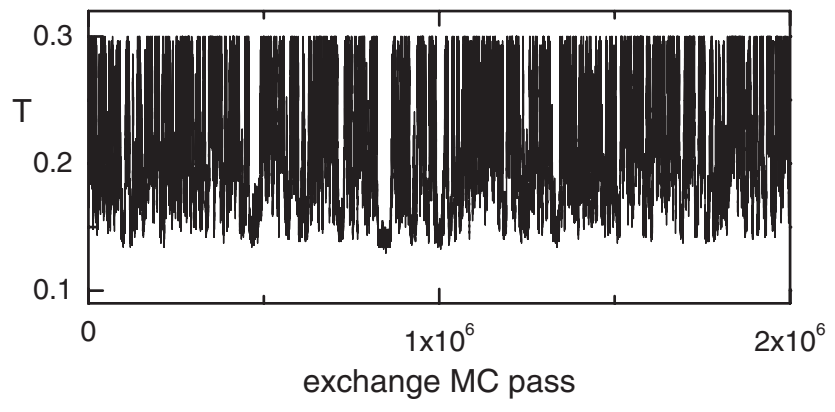

FIG. 1. Trajectory in temperature space of a replica starting at temperature $T=0.30$, for system size $L=55$. The simulation included 100 replicas in the range $T=0.30$ to $T=0.096$. 
trapped in metastable configurations within the available time scale of the present simulation. In fact, below $T_{f}$ the numerical results for $\xi_{\mathrm{ph}}$ and $\xi_{v}$ are sensitive to the initial conditions, while above $T_{f}$ they are not. Our estimate of $T_{f}$ is well below the apparent glass temperature $T_{g}$, observed in earlier MC simulations [7].

For the finite-size scaling analysis of the correlation length $[19,20]$, we consider the dimensionless ratio $\xi / L$, which, for a continuous transition, should satisfy the scaling form

$$
\xi / L=G\left(\left(T-T_{c}\right) L^{1 / \nu}\right),
$$

where $\nu$ is the critical exponent of the power-law divergent correlation length $\xi \propto\left|T-T_{c}\right|^{-\nu}, T_{c}$ is the critical temperature, and $G(x)$ is a scaling function with $G(0)=C$ a constant and $G(x) \rightarrow x^{-\nu}$ as $x \rightarrow \infty$. This scaling form implies that data for the scaled correlation length $\xi / L$ as a function of temperature, for different system sizes $L$, should come together for decreasing temperatures and cross at the same temperature $T=T_{c}$. In addition, the data should splay out for different system sizes with slopes determined by the critical exponent $\nu$.

Figure 2 shows the temperature dependence of the scaled correlation length for phase variables $\xi_{\mathrm{ph}} / L$ in the temperature range where full equilibration was possible and for different system sizes. This quantity increases faster on lowering the temperature as the system size $L$ increases, indicating a divergent length scale for decreasing temperature. However, for fixed temperature it decreases with $L$ even at the lowest available temperature, and therefore the curves do not cross at a common temperature. If a phase-coherence transition takes place, then it should occur at some unknown critical temperature $T_{c}$ much below $T_{f} \sim 0.145$, which is not accessible in our calculations for larger system sizes, or else only at $T=0$.

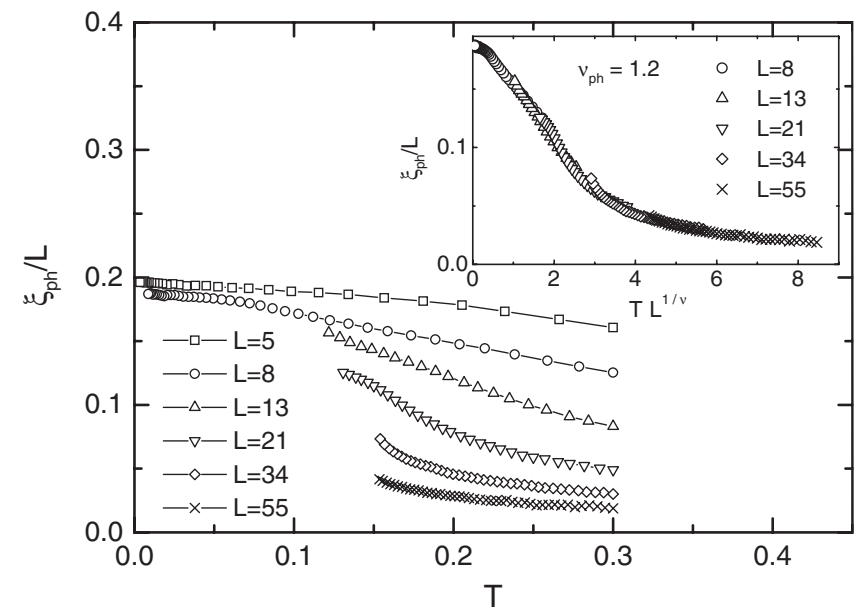

FIG. 2. Scaled correlation length of phase variables $\xi_{\mathrm{ph}} / L$ for different system sizes $L$. Inset: Scaling plot according to Eq. (3), assuming $T_{c}=0$ with $\nu_{\mathrm{ph}}=1.2$.
The latter case corresponds to a transition where $T_{c}=0$ and the correlation length $\xi_{\mathrm{ph}}$ is finite at any nonzero $T$ but diverges when approaching $T=0$. In principle, requiring that the data should satisfy the scaling form of Eq. (3) could be used to determine $T_{c}$ and consequently find out which scenario is realized. However, for $T_{c}>0$ such data collapse needs two different adjustable parameters $T_{c}$ and $\nu$, which is not a sufficiently accurate method. On the other hand, the $T_{c}=0$ scenario can be verified more accurately since the scaling analysis requires adjusting only the critical exponent $\nu$. In this case, the data for $\xi_{\mathrm{ph}}$ should satisfy the finite-size scaling form of Eq. (3) with $T_{c}=0$, and the best data collapse provides an estimate of the critical exponent $\nu_{p h}$. Figure 2 (inset) shows that indeed the data satisfy the scaling form with an exponent $\nu_{\mathrm{ph}}=1.2(2)$.

The $T_{c}=0$ scaling behavior for phase coherence and the exponent $\nu_{\mathrm{ph}}$ are in agreement with results obtained from resistivity scaling using the resistively shuntedjunction model for the dynamics [9] and, more recently, resistivity scaling using a driven MC dynamics [16]. Although $T_{c}=0$, at finite temperatures the relevant divergent correlation length determines both the linear and the nonlinear resistivity of the array leading to a currentvoltage behavior described by the scaling theory. In the present case, where we can define two correlations lengths $\xi_{\text {ph }}$ and $\xi_{v}$, the relevant divergent quantity should be $\xi_{\mathrm{ph}}$ since this is a measure of phase coherence. From the resistivity scaling, the estimate was [16] $\nu_{\mathrm{ph}}=1.4(2)$, which agrees within the errors with the present direct estimate from correlation-length calculations. This quantitative agreement for the value of $\nu_{\mathrm{ph}}$ obtained from equilibrium and dynamical calculations provides strong support for the phase-coherence transition scenario $[9,16]$ with $T_{c}=0$.

In Fig. 3, we show the finite-size behavior of the scaled correlation length for vortex variables $\xi_{v} / L$. This quantity also increases faster on lowering the temperature. For small system sizes $(L=5-13)$ the curves intersect at different temperatures near $T \sim 0.18$, but for larger system sizes they all decrease with $L$ even at the lowest available

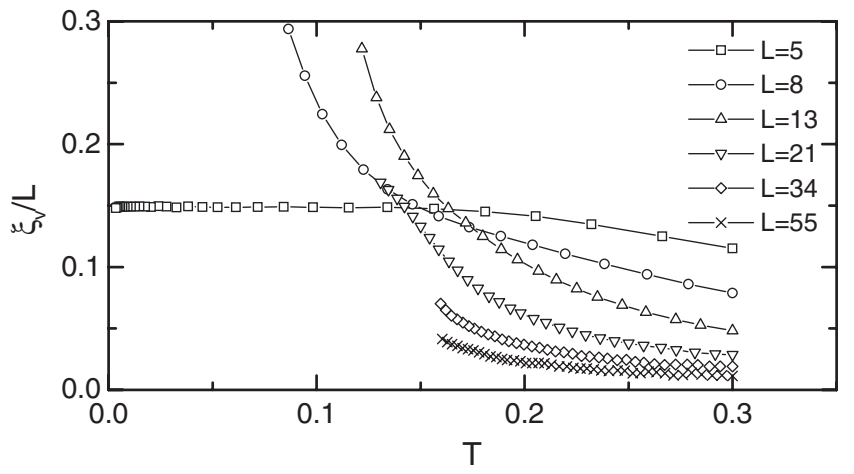

FIG. 3. Scaled correlation length of vortex variables $\xi_{v} / L$ for different system sizes $L$. 


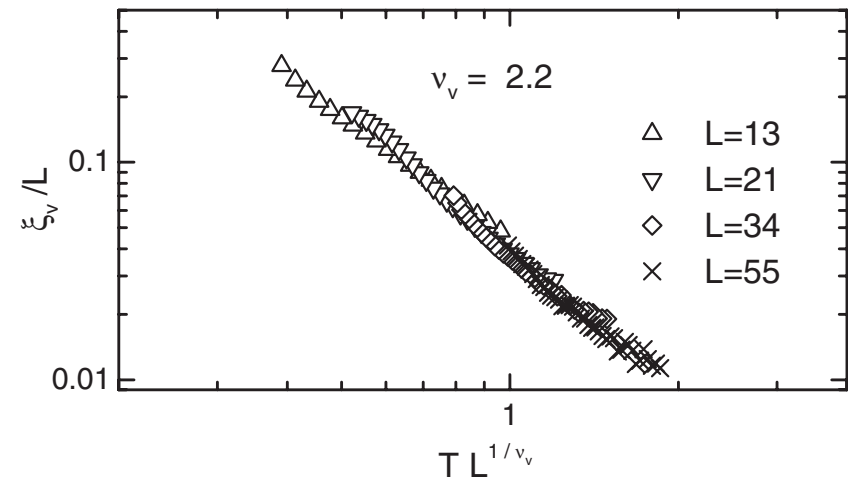

FIG. 4. Scaling plot according to Eq. (3), assuming $T_{c}=0$, for the vortex correlation length $\xi_{v}$, with $\nu_{v}=2.2$.

temperature. Again, the lack of intersection at a common temperature for large system sizes suggests that vortex order, or even vortex-glass order, may occur only at $T_{c}=$ 0 or $T_{c} \ll T_{f}$. Alternatively, this lack of intersection at a common temperature may suggest a vortex first-order transition. Such a transition was proposed earlier based on results from MC simulations in the vortex representation [13], where also a phase-coherence transition at a much lower temperature was observed. The first-order transition was suggested from the observation of a double-peaked energy distribution near the transition. These results are in qualitative agreement with $\mathrm{MC}$ simulations using the phase representation [14]. However, different ground states were found, and the double-peaked energy distribution was also sensitive to the boundary conditions. Moreover, to confirm the first-order nature of the transition, a finite-size scaling analysis of the energy distribution using much larger system sizes would be required. Since the parallel-tempering method used here is known to be a significant improvement over conventional $\mathrm{MC}$ methods by allowing escape from metastable configurations and reducing the equilibration time at low temperatures [18], the lack of full equilibration that we found for $T<T_{f}$ makes unclear whether the double-peaked energy distributions observed in finite systems in other MC simulations $[13,14]$ are the result of an underlying equilibrium first-order transition in the thermodynamic limit or of a (nonequilibrium) freezing transition. Thus, although a first-order vortex transition at finite temperature cannot be ruled out, the possibility remains that this transition is second-order and actually occurs at zero temperature. In this case, the data for $\xi_{v}$ for the largest system sizes should satisfy the finite-size scaling form of Eq. (3) with $T_{c}=0$, and the best data collapse provides an estimate of the critical exponent $\nu_{v}$. Figure 4 shows that indeed the data satisfy this scaling form. Surprisingly, however, the estimated critical exponent for vortex variables $\nu_{v}=2.2(3)$ is significantly different from the one for phase variables $\nu_{\mathrm{ph}}=1.2(2)$. This in turn suggests that the $T_{c}=0$ critical behavior is not described by a single divergent length scale and therefore that there is a decoupling of phase and vortex correlations both diverging as a power law as the temperature approaches zero but with different critical exponents.

It is clear from Figs. 2 and 3 that both correlation lengths $\xi_{\mathrm{ph}}$ and $\xi_{v}$ remain finite at $T \leq T_{g} \sim 0.25$, the apparent glass temperature found in earlier MC simulations [7], since the ratio $\xi / L$ decreases with system size. Therefore, the signature of glass behavior found in this earlier work should be attributed to slow dynamics effects and not an equilibrium phase transition.

In conclusion, our scaling analysis is consistent with a $T_{c}=0$ transition $[6,8,9,16]$, but the phase and vortexcorrelation lengths diverge with different critical exponents suggesting a new decoupled zero-temperature transition scenario.

This work was supported by FAPESP (Grant No. 07/ 08492-9).

[1] J. P. Carini, Phys. Rev. B 38, 63 (1988).

[2] H. S. J. Zant et al., J. Low Temp. Phys. 82, 67 (1991).

[3] I. C. Baek, Y. J. Yun, and M. Y. Choi, Phys. Rev. B 69, 172501 (2004).

[4] F. Yu et al., Phys. Rev. Lett. 68, 2535 (1992).

[5] X. S. Ling et al., Phys. Rev. Lett. 76, 2989 (1996).

[6] S. Teitel and C. Jayaprakash, Phys. Rev. Lett. 51, 1999 (1983).

[7] T. C. Halsey, Phys. Rev. Lett. 55, 1018 (1985).

[8] M. Y. Choi and D. Stroud, Phys. Rev. B 32, 7532 (1985).

[9] E. Granato, Phys. Rev. B 54, R9655 (1996).

[10] R. A. Hyman et al., Phys. Rev. B 51, 15304 (1995).

[11] E. Granato, Phys. Rev. B 58, 11161 (1998).

[12] B. Kim and S. J. Lee, Phys. Rev. Lett. 78, 3709 (1997); M. R. Kolachi and H. Fazli, Phys. Rev. B 62, 9089 (2000); G. Tarjus et al., J. Phys. Condens. Matter 17, R1143 (2005); G. S. Jeon, S. J. Lee, and M. Y. Choi (to be published).

[13] P. Gupta et al., Phys. Rev. Lett. 80, 105 (1998).

[14] C. Denniston and C. Tang, Phys. Rev. B 60, 3163 (1999).

[15] S. Y. Park et al., Phys. Rev. Lett. 85, 3484 (2000).

[16] E. Granato, Phys. Rev. B 75, 184527 (2007).

[17] S. J. Lee et al., Phys. Rev. Lett. 88, 025701 (2001).

[18] K. Hukushima and K. Nemoto, J. Phys. Soc. Jpn. 65, 1604 (1996); E. Marinari and G. Parisi, Europhys. Lett. 19, 451 (1992).

[19] H. G. Ballesteros et al., Phys. Rev. B 62, 14237 (2000).

[20] L. W. Lee and A. P. Young, Phys. Rev. Lett. 90, 227203 (2003).

[21] R. Yamamoto and W. Kob, Phys. Rev. E 61, 5473 (2000).

[22] R. N. Bhatt and A. P. Young, Phys. Rev. B 37, 5606 (1988). 